\title{
Screening of Anticancer Properties of some Medicinal Plants - Review
}

\author{
Jyoti Pande and Sumitra Chanda* \\ Department of Biosciences (UGC-CAS), Saurashtra University, Rajkot360 005, Gujarat, India \\ *Corresponding author
}

\section{A B S T R A C T}

\section{Keywords}

Medicinal plants, anticancer activity, MTT assay, cell lines, in vitro methods

\section{Article Info}

Accepted:

12 February 2020

Available Online:

10 March 2020
Cancer is the most deadly disease in the world and cancer deaths are increasing rapidly in developed and developing countries. There are many types of cancer affecting people of all ages with no discrimination of age and sex. Treatment options are synthetic drugs and naturally derived drugs. The synthetic drugs have many side effects and are not preferred and there is a dire need to have a natural anticancer drug with novel mechanism of action. The plant kingdom with its vast diversity, rich secondary metabolites, easy availability makes them most popular for discovering new anticancer compounds. With this idea, in this review, 50 medicinal plants belonging to 34 families were screened for their anticancer properties. They were used as plant extracts or essential oils or metal nanoparticles. Detailed information is also given regarding the part used, solvent used, assay and cell lines used for evaluating the anticancer properties. These are promising plants and hence potential candidates for further studies which may ultimately lead to new drugs or lead molecules for drug development to be used as natural, novel and safe anticancer agents.

\section{Introduction}

Cancer is a major health hazard both in developed and developing countries and is the second leading cause of death worldwide. It is a frightful and most devastating disease and main cause of morbidity and mortality globally; the number of cases of cancer deaths are increasing rapidly and estimated to be 21 million by 2030 (Siegel et al., 2016). Cancer is a complex disease with more than 100 disorders. It induces abnormal cell growth which spreads to different parts of the body and result in their dysfunction. According to GBD (2015) Disease and Injury Incidence and Prevalence Colloborators (2016) and WHO (2016), 8.8 million deaths occurred due to various types of cancer in 2015. According to Ruckmani et al., (2015), in India 5.5 lakh deaths occur every year and 8 lakh cases are detected.

There are many types of cancer like lung, colon, cervical, prostrate, hepatic, blood, pancreatic, renal, skin, breast but most common are breast, colon, prostrate, and lung 
cancer. Cancer may affect people of all ages but chances increase with age. It is estimated rather statistics indicate that men are prone to lung, colon, rectum and prostate cancer while women are prone to breast, colon, rectal and stomach cancer. Even children below 15 years are prone to cancer mainly because of life style and eating habits (Sirsat et al., 2019).

Treatment options for cancer are chemotherapy, brachytherapy, cryosurgery, hormonal therapy, surgery and chemically derived drugs. Many synthetic anticancer or antitumor drugs are used to treat cancer but there are many side effects like myelosuppression, hair loss, fatigue, infection, etc (Stopeck and Thompson, 2012; Hu et al., 2015).

In fact, chemotherapeutic drugs lead to various side effects while natural anticancer drugs derived from medicinal plant extracts, essential oils or metal nanoparticles selectively induce apoptosis and arrest cancer cells without causing any damage to normal cells (Shahneh et al., 2014).

One of the criterions for an effective and acceptable anti-cancer agent/drug is that it should have no harmful effect on normal cells. Cell cycle arrest of cancer cells is regarded as one of the target mechanisms in cancer treatment (Diaz-Moralli et al., 2013). Various compounds isolated from plants are effective against proliferating cells.

They exhibit cytotoxic effects either by damaging DNA or by blocking the formation of mitotic spindle during different stages of cell division (Gali-Muhtasib and Bakkar, 2002). The need of the hour is to isolate bioactive compounds from medicinal plants that can be a lead molecule for anticancer drug therapy or to develop the crude plant extract itself to become herbal medicine (Singh et al., 2013).
There are a number of anticancer drugs already in use, which are of plant origin. Few examples are vinca alkaloids, vinblastine and vincristine from Catharanthus roseus; Paclitaxol (taxol) from Taxus brevifolia; Himoharringtonine from Cephalotaxus harringtonia; Elliptinium, a derivative of ellipticine isolated from Bleekeria vitensis; Colchicine from Colchium autumnale (Nagani and Chanda, 2013; Iqbal et al., 2017; Seca and Pinto, 2018). There are many medicinal plants which show anticancer properties (Chanda and Nagani, 2013; Sirsat et al., 2019).

Plant compounds with anticancer properties are polyphenols, brassinosteroids and taxols. Polyphenols include flavonoids, tannins, curcurmin, resveratol and gallacatechins. Flavonoids include anthocyanins, flavones, flavonols, chalcones, etc. Two natural brassinosteroids which showed anticancer properties are 28-homocastasterone and 24epibrassinolide (Greenwell and Rahman, 2015).

Plant and plant derived drugs are better alternatives to chemical or synthetic drugs because natural drugs are simple, safe, ecofriendly, economic, less toxic with less side effects ; marine and microbiological organisms have also provided many promising bioactive anticancer compounds for eg. trabectedn, cytotoxic antibiotics of the anthracycline class and enedynes (Amaral et al., 2019).

The anticancer properties of medicinal plant extracts can be evaluated by various in vitro and in vivo models. Some screening in vitro methods for anticancer activity are Tryphan blue dye exclusion assay, Lactate dehydrogenase assay, MTT (3-[4,5dimethylthiazol-2-yl]-2,5-diphenyltetrazolium bromide) assay, XTT (2,3-bis[2-Methoxy-4nitro-5-sulfophenyl]- 2Htetrazolium-5- 
carboxyanilide inner salt ) assay, NRU (Neutral red uptake assay) and SRB (Sulforhodamine B assay); but most popular is MTT assay (Chanda and Nagani, 2013). MTT assay is non-radioactive, quick, simple and affordable method widely used in cytotoxic studies (Russo et al., 2004). Induction of Ehrlich ascites carcinoma in mice represents the in vivo model (Devi et al., 1998).

In MTT assay, different types of cell lines are used for eg. HeLa, PLHC-1, Calu-6 and U251, Ca Ski-, MV-3 (cervical cancer cell lines), T47D, MDA-MB-435S, MDA-MB231 and MCF-7, MCF-12A, Bcap-37, HCC1937 and HCC1143(breast cancer cell lines), L929 (normal fibroblast cancer cell line), HaCaT (human immortalized keratinocyte cells), A-549, Mehr-80, NCI460, , NCI-H460, HOP-6 (lung cancer cell line), HCT-116, HT-29, WiDr, LoVo (colon cancer cell line), CACO-2 ( intestinal cancer cell line), MIAPaCa-2, PANC-1 (pancreatic cancer cell line), MGC-803, ATCC-43504 (human gastric cancer cell line), PA-TU-8902 (pancreas adenocarcinoma cell line), Hep2 (human epiglottis cancer line), WEHI, SAF1, K-562,THP1 (leukemia cancer cell line), HepG2 (hepatocarcinoma cell line), HEK293, 786-0 (human renal cell lines), OVCAR-03, IGR-OV-1 (ovarian cancer cell line), PC-3, DU145, LNCaP ( prostate cancer cell lines), HEK 293, HEK 293T (human kidney cell lines), SMMC-7721 (hepatoma cancer cell line), U251 - (human tumor cell lines) KB, HEp- 2 (nasopharyngeal epidermoid cancer cell line), WISH (human amniotic epithelial cell line), Vero (African green monkey kidney cell line), RAW 264.7 (Murine macrophage cancer cell line), WRL-68 -(normal human hepatic cell line), Jurkat -Human T-cell lymphoma, etc.

The plant extracts, essential oils or nanoparticles are rich in many different phytoconstituants and all work in different manner. Anticancer agents act via many mechanisms. They induce cell cycle arrest and apoptosis; suppress proliferation of cells, inhibit cell cycle progression, inhibit DNA synthesis, rupture plasma membrane, activate caspases, depolarize mitochondria; modulation signal transduction, etc (Saklani et al., 2019). A direct correlation between antioxidant activity and antiproliferative activity is reported by Liu et al., (2002) in Rubus idaeus ; Ghasemzadeh et al., (2018) in Oryza sativa; Wang et al., (2019) in Boehmeria nivea plants.

In the present review, a number of plants, parts and solvents used, cytotoxicity assay and cell lines used, metal nanoparticles used for evaluating cytotoxic potential of medicinal plants is listed (Table 1). Screening of 50 plants was attempted. The 50 plants belonged to 34 different families, in which 8 plants belonged to Lamiaceae family, 6 plants belonged to Fabaceae family, 2 plants belonged to Malvaceae family, 2 plants belonged to Compositae/ Asteraceae family, 2 plants belonged to Moraceae family, 2 plants belonged to Maliaceae family, 2 plants belonged to Caesalpiniaceae family and 2 plants belonged to Rosaceae family.

Different plant extracts showed promising activity against different types of cancer. The parts of the plant used and solvent extracts were also different but they were very effective against a varied number of cancer types. For eg. roots of $R$. cordifolia on kidney, cervical larynx carcinoma (Patel et al., 2011); seeds of $S$. macrophylla on colon carcinoma, nasopharyngeal epidermoid carcinoma, cervical carcinoma and breast carcinoma (Goh and Kadir, 2011); seeds A. heterophyllus embryonic kidney, lung adeno carcinoma, cervical and breast cancer (Patel and Patel 2011); roots of $P$. longipes, $S$. miltiorrhiza, S. sahendica on pancreatic and 
melanoma cancer (Fronza et al., 2011); rhizomes of $A$. mutica on epidermoid, breast, lung, cervical, colon , non-human fibroblast carcinoma (Malek et al., 2011); leaves of $A$. indica on breast adenocarcinoma (Bibi et al., 2012); seeds of $M$. oleifera on lung, liver, colon, neuroblastima (Shaban et al., 2012); muricatan leaves on human hepatic, breast carcinoma and immortalized keratinocyte (George et al., 2012); seeds of $T$. foenum graecum on epidermoid, breast adenocarcinoma (Al-Oqail et al., 2013); leaves of $B$. variegate on ovary, prostrate, lungs, breast, leukemia cancer (Mishra et al., 2013); silver nanoparticles of $C$. guianensis on breast cancer (Devaraj et al., 2013); peel of $H$. polyrhizus and $H$. undatus on prostate, breast and gastric cancer (Luo et al., 2014); fruit kernels of $M$. indica on breast cancer (Abdullah et al., 2014); seeds of N. sativa on lung cancer (Al-Sheddi et al., 2014); Mistletoe of $V$. album on breast carcinoma, pancreas adenocarcinoma, prostate carcinoma, lung carcinoma (Weissenstein et al., 2014); seeds of $P$. cubeba on breast cancer (Graidist et al., 2015); G. glabra, P. lactiflora, and E. japonica on murine macrophage (Zhou et al., 2015); zinc oxide nanoparticles of $D$. regia on lung cancer (Sathyabama and Sankaranarayanan, 2015); gold nanoparticles of $T$. terrestris on adenocarcinoma (Gopinath et al., 2016); wood of $L$. amara on cervical and breast cancer (Zubair et al., 2016); fruits of $O$. bacaba on breast cancer (Finco et al., 2016); bark and leaf of $P$. eldarica on cervical and breast cancer (Sarvmeili et al., 2016); A. odorata leaves on breast adenocarcinoma (Boutennoun et al., 2017); aerial parts of $O$. vulgare on hepatocellular carcinoma and embryonic kidney (Elshafie et al., 2017); M. nigra fruit on prostate adenocarcinoma (Turan et al., 2017); O. vulgare leaves on kidney leucocyte and tumor (Beltrán et al., 2017); aerial parts of D. kotschyi on pulmonary adenocarcinoma and lung cancer (Sani et al.,
2017); methanol extract of leaf, stem and bark of Pterocarpus santalinus on human cervical cancer (Donga et al., 2017), Leea indica leaves on human prostate cancer (Ghagane et al., 2017), root bark of Crataeva magna on Ehrlich ascites carcinoma (Meera and Chidambaranathan, 2017), aerial parts of $T$. vulgaris on human tumor, colon, intestinal and breast carcinomas (Hassan et al., 2018); fruits of $R$. canina on colon cancer (Turan et al., 2018); whole plant of $S$. barbata on hepatoma, colon and breast cancer (Wang et al., 2018); leaves and stem of T. hypoleuca on melanoma, breast, kidney, lung, prostate, ovary, colon and leukemia cancer (Perera et al., 2019); leaves, fruits and seeds of $A$. obesum on breast cancer (Ali et al., 2019); leaves of $C$. sativus on breast and cervical cancer (Tuama and Mohammed, 2019); bark of A. lebbeck on breast cancer (Sivaraj et al., 2019); silver nanoparticles of M. umbellatum on breast cancer (AlSalhi et al., 2019); aerial parts of $S$. officinalis on prostate, breast and cervical cancer (Privitera et al., 2019); gold nanoparticles of $S$. barbata on pancreatic cancer (Wang et al., 2019); copper nanoparticle of $T$. japonica on colon, hepatic and breast cancer (Hassanien et al., 2019); copper nanoparticle of $S$. alternifolium on breast cancer (Yugandha et al., 2019), leaves of Aloe castellorum and Aloe pseudorubroviolacea on colon carcinomas (Ahamed et al., 2020); aerial parts of Azadirachta indica and Melia azedarach on breast cancer (Malar et al., 2020 ); silver nanoparticles of Tamarindus indica on breast cancer (Gomathi et al., 2020); whole plants of Rumex vesicarius on breast, colon and liver carcinoma (Farooq et al., 2020), etc.

The promising activity of these plants as anticancer agents is because of the secondary metabolites present in them. The secondary metabolites may be alkaloids, flavonoids, phenols, tannins, terpenoids, anthraquinones, saponins and may be obtained from any part 
of the plant and not restricted to any particular plant organ. The secondary metabolites may be present in plant aqueous extract, or organic solvent extracts, essential oils, or nano particles synthesized using any plant part (Sirsat et al., 2019) . Phenols and flavonoids derived from medicinal plants showed anticancer properties (Gibellini et al., 2010; Mavundza et al., 2010). Anti cancer property of A. indica and $M$. azedarach extracts is reported by Malar et al., (2020). Both these plants showed the presence of secondary metabolites like flavonoids, phenols, steroids, alkaloids, tannins, saponins, anthraquinones, etc. Different medicinal plants listed in Table 1 showed anticancer properties and it is attributed for the presence of various phytochemicals in them.

For eg. n- hexane extract of $P$. longipes contained 7a-acetoxyroyleanone, horminone, royleanone, 7-ketoroyleanone, 7aethoxyroyleanone, iguestol, deoxyneocryptotanshinone, 12-hydroxy-11methoxyabieta-8,11,13-trien-7-one,

inuroyleanol, sugiol, cryptojapanol, orthosiphonol; n- hexane extract $S$. miltiorrhiza contained tanshinone, cryptotanshinone, tanshinone i, 1,2dihydrotanshinone, miltirone, 1-oxomiltirone, miltiodiol, ferruginol, sahandinone, camptothecin (Fronza et al., 2011); ethyl acetate extract of A. mutica contained flavokawin B, 5,6-dehydrokawain, alpinetin, pinostrobin chalcone (Malek et al., 2011); nbutanolic leaf extract of Annona muricata contained flavonols, polyphenols and flavones (George et al., 2012); different solvent extracts of $B$. variegata contained terpenoids, phenolics, flavonoids, anthraquinones, saponins, tannins, alkaloids (Mishra et al., 2013); supercritical carbon dioxide extracts of pitaya $H$. polyrhizus and $H$. undatus peel contained $\beta$-amyrin, $\beta$-sitosterol, and stigmast-4-en-3-one, octadecane, 1- tetracosanol, Heptacosane, campesterol, nonacosane, trichloroacetic acid, hexadecyl ester (Luo et al., 2014); methanol extract of $P$. cubeba contained monoterpenes, sesquiterpenes, $\beta$-elemene, $\beta$-cubebene, $\beta$ pinene (Graidist et al., 2015) ; ethanolic extract of Mangifera indica contained phenol, 4,6-di (1,1-dimethylethyl)-2-methyl (Abdullah et al., 2014); methanolic extract of L. amara contained alkaloids and lunacrine (Zubair et al., 2016); ethanolic extract of Cratavea magna contained flavonoids, alkaloids and tannins (Meera and Chidambaranathan, 2017); aqueous and ethanolic extracts of $O$. vulgare contained phenolic compounds, flavonoids, chlorogenic, caffeic, pcoumaric, ferulic, rosmarinic and ursolic acids p-cymene, 1-octocosanol and phytol (Beltrán et al., 2017); dimethyl sulfoxide extract of $M$. nigra contained ascorbic acid, gallic acid , 3,4-dihydroxy benzoic acid, protocatechuic acid, chlorogenic acid, caffeic acid, epigallocatechin gallate, pcoumaric acid, rutinhydrate (Turan et al., 2017); essential oils of $O$. vulgare contained limonene, thymol, carvacrol ,citral (Elshafie et al., 2017 ); essential oil of T. vulgaris contained P-cymene, $\gamma$ terpenine, thymyl methyl ether, thymol, p-cymene, o-cymene (Hassan et al., 2018), etc. different solvent extracts from $T$. rosea contained o-xylene, 2,4-dimethylhexane, methyl cyclohexane, methylbenzene, 3-Pentene-2-one, alkaloid and pentacyclic triterpenes (Perera et al., 2019); methanol bark extract of A. lebbeck contained 2-(4-methyloctadecanoyl)imidazole, levo-5adihydronorgestrel, 9-octadecyonoic acid, methyl ester, octadec-9-enoic acid, 10octadecenoic acid, methyl ester, dodecanoic acid, 11-oxo-methyl ester, 4,7methanoazulene, decahydro - 1,4,9,9tetramethyl, benzene, 1-pentynyl, benzene, 1,4-bis(4-acetylphenyliminomethyl), 4h1benzopyron-4-one,7-hydroxy (Sivaraj et al., 2019), etc. 
Table.1 List of medicinal plants, their family, parts, solvents used for extraction, assay and cell line employed for cytotoxicity studies

\begin{tabular}{|c|c|c|c|c|c|}
\hline $\begin{array}{l}\text { Sr. } \\
\text { No }\end{array}$ & Botanical name (family) & $\begin{array}{l}\text { Plant } \\
\text { part }\end{array}$ & $\begin{array}{l}\text { Solvent / } \\
\text { Essential oil/ } \\
\text { Nanoparticle }\end{array}$ & Assay/ Cell line & References \\
\hline 1 & $\begin{array}{l}\text { Abelmoschus esculentus } \\
\text { L. } \\
\text { (Malvaceae) }\end{array}$ & $\begin{array}{l}\text { fruit } \\
\text { pulp }\end{array}$ & $\begin{array}{l}\text { silver } \\
\text { nanoparticles }\end{array}$ & $\begin{array}{l}\text { MTT assay- } \\
\text { Jurkat cell line }\end{array}$ & $\begin{array}{l}\text { Mollick et al., } \\
2015\end{array}$ \\
\hline 2 & $\begin{array}{l}\text { Achillea odorata L. } \\
\text { (Asteraceae) }\end{array}$ & leaf & methanol & $\begin{array}{l}\text { MTT assay- } \\
\text { MCF-7, Hep2, WEHI }\end{array}$ & $\begin{array}{l}\text { Boutennoun et } \\
\text { al., } 2017\end{array}$ \\
\hline 3 & $\begin{array}{l}\text { Adenium obesum (Forssk.) } \\
\text { Roem. \& } \\
\text { Schult.(Apocynaceae) }\end{array}$ & $\begin{array}{l}\text { leaf, } \\
\text { fruit, } \\
\text { seed }\end{array}$ & $\begin{array}{l}95 \% \text { ethanol, } \\
\text { aqueous }\end{array}$ & $\begin{array}{l}\text { MTT assay- } \\
\text { MCF-7 }\end{array}$ & Ali et al., 2019 \\
\hline 4 & $\begin{array}{l}\text { Aesculus indica } \mathrm{L} \text {. } \\
\text { (Sapindaceae) }\end{array}$ & leaf & $\begin{array}{l}\text { methanol, } \\
\text { aqueous }\end{array}$ & $\begin{array}{l}\text { MTT assay- } \\
\text { MCF-7 }\end{array}$ & Bibi et al., 2012 \\
\hline 5 & $\begin{array}{l}\text { Albizia lebbeck L. } \\
\text { (Fabaceae) }\end{array}$ & bark & methanol & $\begin{array}{l}\text { MTT assay- } \\
\text { MCF-7- }\end{array}$ & $\begin{array}{l}\text { Sivaraj et al., } \\
2019\end{array}$ \\
\hline 6 & $\begin{array}{l}\text { Aloe castellorum } \\
\text { J.R.I.Wood } \\
\text { Aloe pseudorubroviolacea } \\
\text { L. (Asphodelaceae) }\end{array}$ & leaf & methanol & $\begin{array}{l}\text { MTT assay- } \\
\text { HCT-116 }\end{array}$ & $\begin{array}{l}\text { Ahamed et al., } \\
2020\end{array}$ \\
\hline 7 & $\begin{array}{l}\text { Alpinia mutica Roxb. } \\
\text { (Zingiberaceae) }\end{array}$ & rhizome & $\begin{array}{l}\text { methanol, } \\
\text { hexane, } \\
\text { ethyl acetate, } \\
\text { aqueous }\end{array}$ & $\begin{array}{l}\text { (NRU) Neutral red } \\
\text { uptake assay- } \\
\text { KB,MCF7,A549 } \\
\text { Ca Ski, HCT116, } \\
\text { HT29,MRC5 }\end{array}$ & $\begin{array}{l}\text { Malek et al., } \\
2011\end{array}$ \\
\hline 8 & $\begin{array}{l}\text { Annona muricata L. } \\
\text { (Annonaceae) }\end{array}$ & leaf & n-butanol & $\begin{array}{l}\text { XTT assay- } \\
\text { WRL-68, } \\
\text { MDA-MB-435S, } \\
\text { HaCaT }\end{array}$ & $\begin{array}{l}\text { George } \text { et al., } \\
2012\end{array}$ \\
\hline 9 & $\begin{array}{l}\text { Artocarpus heterophyllus } \\
\text { Lam. } \\
\text { (Moraceae) }\end{array}$ & seed & methanol & $\begin{array}{l}\text { MTT assay- } \\
\text { HEK 293T, A549, } \\
\text { HeLa, MCF-7 }\end{array}$ & $\begin{array}{l}\text { Patel and Patel } \\
2011\end{array}$ \\
\hline 10 & $\begin{array}{l}\text { Azadirachta indica A. } \\
\text { Juss } \\
\text { Melia azedarach } \mathrm{L} . \\
\text { (Meliaceae) }\end{array}$ & $\begin{array}{l}\text { aerial } \\
\text { parts }\end{array}$ & $\begin{array}{l}\text { petroleum ether, } \\
\text { methanol, } \\
\text { hexane, } \\
\text { aqueous }\end{array}$ & $\begin{array}{l}\text { MTT assay- } \\
\text { MCF-7 }\end{array}$ & $\begin{array}{l}\text { Malar et al., } \\
2020\end{array}$ \\
\hline 11 & $\begin{array}{l}\text { Bauhinia variegate } \\
\text { L. (Leguminosae/ } \\
\text { Fabaceae) }\end{array}$ & leaf & $\begin{array}{l}\text { petroleum ether, } \\
\text { benzene, } \\
\text { chloroform, } \\
\text { ethyl acetate, } \\
\text { acetone, } \\
\text { ethanol, } \\
\text { aqueous }\end{array}$ & $\begin{array}{l}\text { SRB assay- } \\
\text { IGR-OV-1, DU-145, } \\
\text { HOP-6, MCF-7, THP1 }\end{array}$ & $\begin{array}{l}\text { Mishra et al., } \\
2013\end{array}$ \\
\hline
\end{tabular}




\begin{tabular}{|c|c|c|c|c|c|}
\hline 12 & $\begin{array}{l}\text { Caesalpinia pulcherrima } \\
\text { L. (Caesalpiniaceae) }\end{array}$ & flower & $\begin{array}{l}\text { silver } \\
\text { nanoparticles }\end{array}$ & $\begin{array}{l}\text { MTT assay } \\
\text { HeLa }\end{array}$ & $\begin{array}{l}\text { Moteriya and } \\
\text { Chanda, } 2017\end{array}$ \\
\hline 13 & $\begin{array}{l}\text { Couroupita guianensis } \\
\text { Aubl. (Lecythidaceae) }\end{array}$ & leaf & $\begin{array}{l}\text { silver } \\
\text { nanoparticles }\end{array}$ & $\begin{array}{l}\text { MTT assay- } \\
\text { MCF-7 }\end{array}$ & $\begin{array}{l}\text { Devaraj et al., } \\
2013\end{array}$ \\
\hline 14 & $\begin{array}{l}\text { Cucumis sativus L. } \\
\text { (Cucurbitaceae) }\end{array}$ & leaf & $\begin{array}{l}\text { methanol, } \\
\text { acetone }\end{array}$ & $\begin{array}{l}\text { MTT assay- } \\
\text { MCF 7, HeLa }\end{array}$ & $\begin{array}{l}\text { Tuama and } \\
\text { Mohammed, } \\
2019\end{array}$ \\
\hline 15 & $\begin{array}{l}\text { Delonix regia } \mathrm{L} . \\
\text { (Caesalpiniaceae) }\end{array}$ & flower & $\begin{array}{l}\text { zinc oxide } \\
\text { nanoparticles }\end{array}$ & $\begin{array}{l}\text { MTT assay- } \\
\text { A549 }\end{array}$ & $\begin{array}{l}\text { Sathyabama and } \\
\text { Sankaranarayana } \\
\mathrm{n}, 2015\end{array}$ \\
\hline 16 & $\begin{array}{l}\text { Dracocephalum kotschyi } \\
\text { Boiss. (Lamiaceae) }\end{array}$ & $\begin{array}{l}\text { aerial } \\
\text { part }\end{array}$ & $\begin{array}{l}\text { methanol, } \\
\text { dichloromethane, } \\
\text { ethyl acetate, } \\
\text { hexane, } \\
\text { aqueous, } \\
\text { essential oil }\end{array}$ & $\begin{array}{l}\text { MTT assay- } \\
\text { Calu-6, Mehr-80, L929 }\end{array}$ & Sani et al., 2017 \\
\hline 17 & $\begin{array}{l}\text { Glycyrrhiza glabra } \\
\text { L.(Fabaceae) } \\
\text { Paeonia lactiflora } \\
\text { Pall.(Paeoniaceae) } \\
\text { Eriobotrya japonica } \\
\text { (Thunb.) Lindl (Rosaceae) }\end{array}$ & - & $\begin{array}{l}\text { methanol, } \\
50 \% \text { ethanol, } \\
96 \% \text { ethanol, }\end{array}$ & $\begin{array}{l}\text { MTT assay- } \\
\text { RAW } 264.7\end{array}$ & $\begin{array}{l}\text { Zhou et al., } \\
2015\end{array}$ \\
\hline 18 & $\begin{array}{l}\text { Hylocereus polyrhizus } \\
\text { Weber. } \\
\text { Hylocereus undatus } \\
\text { Haworth.(Cactoideae) }\end{array}$ & peel & $\begin{array}{l}\text { supercritical } \\
\text { carbondioxide }\end{array}$ & $\begin{array}{l}\text { MTT assay- } \\
\text { PC3,Bcap-37, } \\
\text { MGC-803 }\end{array}$ & Luo et al., 2014 \\
\hline 19 & $\begin{array}{l}\text { Lunasia amara Blanco. } \\
\text { (Rutaceae) }\end{array}$ & wood & $\begin{array}{l}\text { methanol, } \\
\text { ethyl acetate, } \\
\text { n-hexane }\end{array}$ & $\begin{array}{l}\text { MTT assay- } \\
\text { HeLa, T47D }\end{array}$ & $\begin{array}{l}\text { Zubair et al., } \\
2016\end{array}$ \\
\hline 20 & $\begin{array}{l}\text { Mangifera indica } \mathrm{L} . \\
\text { (Anacardiaceae) }\end{array}$ & $\begin{array}{l}\text { Fruit } \\
\text { kernel }\end{array}$ & ethanol & $\begin{array}{l}\text { MTT assay- } \\
\text { MDA-MB-231and } \\
\text { MCF-7, MFC-10A }\end{array}$ & $\begin{array}{l}\text { Abdullah et al., } \\
2014\end{array}$ \\
\hline 21 & $\begin{array}{l}\text { Memecylon umbellatum } \\
\text { Burm F. } \\
\text { (Melastomataceae) }\end{array}$ & leaf & $\begin{array}{l}\text { silver } \\
\text { nanoparticles }\end{array}$ & $\begin{array}{l}\text { MTT assay- } \\
\text { MCF-7 }\end{array}$ & $\begin{array}{l}\text { AlSalhi et al., } \\
2019\end{array}$ \\
\hline 22 & $\begin{array}{l}\text { Moringa oleifera Lam. } \\
\text { (Moringaceae) }\end{array}$ & seed & methanol & $\begin{array}{l}\text { SRB assay- } \\
\text { A-549, Hep-2, } \\
\text { HT-29, IMR-32 }\end{array}$ & $\begin{array}{l}\text { Shaban et al., } \\
2012\end{array}$ \\
\hline 23 & $\begin{array}{l}\text { Morus nigra } \mathrm{L} \text {. } \\
\text { ( Moraceae) }\end{array}$ & fruit & $\begin{array}{l}\text { dimethyl } \\
\text { sulfoxide }\end{array}$ & $\begin{array}{l}\text { MTT assay- } \\
\text { PC-3 }\end{array}$ & $\begin{array}{l}\text { Turan et al., } \\
2017\end{array}$ \\
\hline 24 & $\begin{array}{l}\text { Nigella sativa } \mathrm{L} . \\
\text { (Ranunculaceae) }\end{array}$ & seed & $\begin{array}{l}\text { essential oil, } \\
\text { ethanol }\end{array}$ & $\begin{array}{l}\text { MTT assay- } \\
\text { A-549 }\end{array}$ & $\begin{array}{l}\text { Al-Sheddi et al., } \\
2014\end{array}$ \\
\hline 25 & $\begin{array}{l}\text { Oenocarpus bacaba } \\
\text { Mart.(Arecaceae) }\end{array}$ & fruit & $80 \%$ acetone & $\begin{array}{l}\text { Methyle blue assay- } \\
\text { MCF-7 }\end{array}$ & $\begin{array}{l}\text { Finco et al., } \\
2016\end{array}$ \\
\hline 26 & Origanum vulgare L. & aerial & essential oils & MTT assay- & Elshafie et al., \\
\hline
\end{tabular}




\begin{tabular}{|c|c|c|c|c|c|}
\hline & (Lamiaceae) & part & & HepG2, HEK293 & 2017 \\
\hline 27 & $\begin{array}{l}\text { Origanum vulgare } \mathrm{L} . \\
\text { (Lamiaceae) }\end{array}$ & leaf & aqueous ethanol & $\begin{array}{l}\text { MTT assay- } \\
\text { SAF-1, PLHC-1 }\end{array}$ & $\begin{array}{l}\text { Beltrán et al., } \\
2018\end{array}$ \\
\hline 28 & $\begin{array}{l}\text { Peltodon longipes Benth. } \\
\text { Salvia miltiorrhiza } \mathrm{L} . \\
\text { Salvia sahendica } \mathrm{L} . \\
\text { (Lamiaceae) }\end{array}$ & root & n-hexane & $\begin{array}{l}\text { MTT assay- } \\
\text { MIAPaCa-2, MV-3 }\end{array}$ & $\begin{array}{l}\text { Fronza et al., } \\
2011\end{array}$ \\
\hline 29 & $\begin{array}{l}\text { Peltophorum pterocarpum } \\
\text { (DC.) } \\
\text { (Fabaceae) }\end{array}$ & flower & $\begin{array}{l}\text { zinc oxide } \\
\text { nanoparticles }\end{array}$ & $\begin{array}{l}\text { MTT assay } \\
\text { HeLa }\end{array}$ & $\begin{array}{l}\text { Khara et al., } \\
2018\end{array}$ \\
\hline 30 & $\begin{array}{l}\text { Pinus eldarica L. } \\
\text { (Pinaceae) }\end{array}$ & $\begin{array}{l}\text { bark, } \\
\text { leaf }\end{array}$ & $\begin{array}{l}\text { essential oil, } \\
70 \% \text { methanol }\end{array}$ & $\begin{array}{l}\text { MTT assay- } \\
\text { HeLa, MCF-7 }\end{array}$ & $\begin{array}{l}\text { Sarvmeili et al., } \\
2016\end{array}$ \\
\hline 31 & $\begin{array}{l}\text { Piper cubeba L. } \\
\text { (Piperaceae) }\end{array}$ & seed & methanol & $\begin{array}{l}\text { MTT assay- } \\
\text { L929, } \\
\text { MCF-12A, } \\
\text { MCF-7,MDA-MB-468, } \\
\text { MDA-MB-231 }\end{array}$ & $\begin{array}{l}\text { Graidist et al., } \\
2015\end{array}$ \\
\hline 32 & $\begin{array}{l}\text { Rhododendron arboretum } \\
\text { Sm. } \\
\text { (Ericaceae) }\end{array}$ & $\begin{array}{l}\text { leaf, } \\
\text { flower }\end{array}$ & methanol & $\begin{array}{l}\text { MTT assay } \\
\text { HeLa, } \\
\text { MCF-7, A549 }\end{array}$ & $\begin{array}{l}\text { Gautam et al., } \\
2020\end{array}$ \\
\hline 33 & $\begin{array}{l}\text { Rosa canina } \mathrm{L} \text {. } \\
\text { (Rosaceae) }\end{array}$ & fruit & $\begin{array}{l}\text { dimethyl } \\
\text { sulfoxide }\end{array}$ & $\begin{array}{l}\text { MTT assay- } \\
\text { WiDr }\end{array}$ & $\begin{array}{l}\text { Turan et al., } \\
2018\end{array}$ \\
\hline 34 & $\begin{array}{l}\text { Rubia cordifolia L. } \\
\text { (Rubiaceae) }\end{array}$ & root & $\begin{array}{l}\text { methanol, } \\
\text { petroleum ether, } \\
\text { dichloromethane }\end{array}$ & $\begin{array}{l}\text { XTT assay- } \\
\text { HEK 293, HeLa, } \\
\text { HEp-2 }\end{array}$ & $\begin{array}{l}\text { Patel et al., } \\
2011\end{array}$ \\
\hline 35 & $\begin{array}{l}\text { Ruellia britoniana } \mathrm{L} \text {. } \\
\text { (Acanthaceae) }\end{array}$ & flower & $\begin{array}{l}\text { n-hexane, } \\
\text { ethyl acetate, } \\
\text { ethanol }\end{array}$ & $\begin{array}{l}\text { MTT assay } \\
\text { HeLa }\end{array}$ & $\begin{array}{l}\text { Tejaputri et al., } \\
2020\end{array}$ \\
\hline 36 & $\begin{array}{l}\text { Rumex vesicarius } \mathrm{L} . \\
\text { (Polygonaceae) }\end{array}$ & $\begin{array}{l}\text { whole } \\
\text { plant }\end{array}$ & $\begin{array}{l}\text { methanol, } \\
\text { chloroform, } \\
\text { hexane, } \\
\text { ethyl acetate }\end{array}$ & $\begin{array}{l}\text { MTT assay- } \\
\text { MCF-7, } \\
\text { LoVo, Caco-2, } \\
\text { HepG2 }\end{array}$ & $\begin{array}{l}\text { Farooq et al., } \\
2020\end{array}$ \\
\hline 37 & $\begin{array}{l}\text { Salvia officinalis } \mathrm{L} . \\
\text { (Lamiaceae) }\end{array}$ & $\begin{array}{l}\text { aerial } \\
\text { part }\end{array}$ & essential oil & $\begin{array}{l}\text { MTT assay- } \\
\text { LNCaP, MCF-7, } \\
\text { HeLa }\end{array}$ & $\begin{array}{l}\text { Privitera et al., } \\
2019\end{array}$ \\
\hline 38 & $\begin{array}{l}\text { Scutellaria barbata D.Don } \\
\text { (Lamiaceae) }\end{array}$ & $\begin{array}{l}\text { whole } \\
\text { plant }\end{array}$ & acetone & $\begin{array}{l}\text { MTT assay- } \\
\text { LoVo,SMMC-7721, } \\
\text { HCT-116, MCF-7 }\end{array}$ & $\begin{array}{l}\text { Wang et al., } \\
2018\end{array}$ \\
\hline 39 & $\begin{array}{l}\text { Scutellaria barbata } \\
\text { L.(Lamiaceae) }\end{array}$ & $\begin{array}{l}\text { whole } \\
\text { plant }\end{array}$ & $\begin{array}{l}\text { gold } \\
\text { nanoparticles }\end{array}$ & $\begin{array}{l}\text { MTT assay- } \\
\text { PANC-1 }\end{array}$ & $\begin{array}{l}\text { Wang et al., } \\
2019\end{array}$ \\
\hline 40 & $\begin{array}{l}\text { Swietenia macrophylla } \\
\text { King. (Meliaceae) }\end{array}$ & seed & ethanol & $\begin{array}{l}\text { MTT assay- } \\
\text { HCT } 116, \mathrm{~KB}, \\
\text { Ca Ski, MCF-7 }\end{array}$ & $\begin{array}{l}\text { Goh and Kadir, } \\
2011\end{array}$ \\
\hline 41 & $\begin{array}{l}\text { Syzygium alternifolium } \\
\text { (Wt.) }\end{array}$ & $\begin{array}{l}\text { stem, } \\
\text { bark }\end{array}$ & $\begin{array}{l}\text { copper } \\
\text { nanoparticles }\end{array}$ & $\begin{array}{l}\text { MTT assay- } \\
\text { MDA-MB-231 }\end{array}$ & $\begin{array}{l}\text { Yugandhar et } \\
\text { al., } 2019\end{array}$ \\
\hline
\end{tabular}




\begin{tabular}{|c|c|c|c|c|c|}
\hline & (Myrtaceae) & & & & \\
\hline 42 & $\begin{array}{l}\text { Tabebuia hypoleuca (C. } \\
\text { Wright) Urb. } \\
\text { (Bignoniaceae) }\end{array}$ & $\begin{array}{l}\text { leaf, } \\
\text { stem }\end{array}$ & $\begin{array}{l}\text { n-hexane, } \\
\text { ethyl acetate, } \\
\text { methanol }\end{array}$ & $\begin{array}{l}\text { MTT assay- } \\
\text { U251, MCF-7, NCI- } \\
\text { 460, OVCAR-03, PC- } \\
\text { 3, HT-29, 786-0, } \\
\text { K-562 }\end{array}$ & $\begin{array}{l}\text { Perera et al., } \\
2019\end{array}$ \\
\hline 43 & $\begin{array}{l}\text { Tamarindus indica } \mathrm{L} \text {. } \\
\text { (Fabaceae) }\end{array}$ & $\begin{array}{l}\text { fruit } \\
\text { shell }\end{array}$ & $\begin{array}{l}\text { silver } \\
\text { nanoparticles }\end{array}$ & $\begin{array}{l}\text { MTT assay } \\
\text { MCF-7 }\end{array}$ & $\begin{array}{l}\text { Gomathi et al., } \\
2020\end{array}$ \\
\hline 44 & $\begin{array}{l}\text { Thymus vulgaris } \mathrm{L} . \\
\text { (Lamiaceae) }\end{array}$ & $\begin{array}{l}\text { aerial } \\
\text { part }\end{array}$ & essential oils & $\begin{array}{l}\text { A-549, HCT-116, } \\
\text { CACO-2, MCF-7 }\end{array}$ & $\begin{array}{l}\text { Hassan et al., } \\
2018\end{array}$ \\
\hline 45 & $\begin{array}{l}\text { Tilia japonica L. } \\
\text { (Malvaceae) }\end{array}$ & leaf & $\begin{array}{l}\text { copper } \\
\text { nanoparticles }\end{array}$ & $\begin{array}{l}\text { MTT assay- } \\
\text { CACO -2, HepG2, } \\
\text { MCF- } 7\end{array}$ & $\begin{array}{l}\text { Hassanien } \text { et al., } \\
2019 \text { c39 }\end{array}$ \\
\hline 46 & $\begin{array}{l}\text { Tribulus terrestris L. } \\
\text { (Zygophyllaceae) }\end{array}$ & fruit & $\begin{array}{l}\text { gold } \\
\text { nanoparticles }\end{array}$ & $\begin{array}{l}\text { MTT assay- } \\
\text { ATCC-43504 }\end{array}$ & $\begin{array}{l}\text { Gopinath et al., } \\
2016\end{array}$ \\
\hline 47 & $\begin{array}{l}\text { Tridax procumbens } \mathrm{L} . \\
\text { (Compositae) }\end{array}$ & leaf & $\begin{array}{l}\text { Methanol, } \\
\text { ethanol, } \\
\text { aqueous, } \\
\text { chloroform, } \\
\text { acetone, } \\
\text { ethyl acetate }\end{array}$ & $\begin{array}{l}\text { MTT assay- } \\
\text { A549, MCF-7 }\end{array}$ & Syed et al., 2020 \\
\hline 48 & $\begin{array}{l}\text { Trigonella foenum } \\
\text { graecum L. (Fabaceae) }\end{array}$ & seed & essential oil & $\begin{array}{l}\text { MTT and NRU assay - } \\
\text { HEp2, MCF-7,WISH, } \\
\text { Vero }\end{array}$ & $\begin{array}{l}\text { Al-Oqail et al., } \\
2013\end{array}$ \\
\hline 49 & $\begin{array}{l}\text { Viscum album L. } \\
\text { (Santalaceae) }\end{array}$ & $\begin{array}{l}\text { mistleto } \\
\text { e }\end{array}$ & aqueous & $\begin{array}{l}\text { MTT assay- } \\
\text { HCC1937and HCC114, } \\
\text { PA-TU-8902, } \\
\text { DU145, NCI-H460 }\end{array}$ & $\begin{array}{l}\text { Weissenstein et } \\
\text { al., } 2014\end{array}$ \\
\hline 50 & $\begin{array}{l}\text { Ziziphus nummularia } \\
\text { Burm.f. (Rhamnaceae) }\end{array}$ & leaf & $\begin{array}{l}\text { zinc oxide } \\
\text { nanoparticles }\end{array}$ & $\begin{array}{l}\text { MTT assay- } \\
\text { HeLa }\end{array}$ & $\begin{array}{l}\text { Padalia and } \\
\text { Chanda, } 2017\end{array}$ \\
\hline
\end{tabular}

Other than plant aqueous or solvent extracts, essential oils and nano particles synthesized using plant extract also showed anticancer properties. Some essential oils showing anticancer activity are Cinnamomum cassia (Chang et al., 2017); Citrus sinensis (Yang et al., 2017); Rhizoma Curcumae (Zhong et al., 2018); Prunus cerasus cerry (Maragheh et al., 2019), etc. Some of the examples of metal nanoparticles showing anticancer property using different plant parts are silver nanoparticles synthesized using seed extract of Alpinia katsumadai (He et al., 2017), latex of Euphorbia antiquorum L. (Rajkuberan et al., 2017), leaf extract of Cynara scolymus
(Erdogan et al., 2019), pulp extract of Abelmoschus esculentus (Mollick et al., 2019). Gold nanoparticles synthesized from peel extract of Citrus maxima (Yuan et al., 2017), Guazuma ulmifolia barksynthesized $\mathrm{Ag}, \mathrm{Au}$ and $\mathrm{Ag} / \mathrm{Au}$ alloy nanoparticles (Karthika et al., 2017), plant extract of Scutellaria barbata (Wang et al., 2019), rhizome of Zingiber officinale (Ascar et al., 2019).

Zinc oxide nanopartciles synthesized using flower extract of Nyctanthes arbortristis (Jamdagni et al., 2018), fruit extract of Vaccinium arctostaphylos (Mohammadi- 
Aloucheh et al., 2018), root extract of Scutellaria baicalensis (Chen et al., 2019).

This review summarizes some selected medicinal plants showing anticancer properties. In vitro studies have been done with promising results so they can be exploited for plant based anticancer drugs in the near future.

However, detailed studies have to be done on the structural characterization of the phytochemicals involved and their molecular mechanism of action has to be worked out especially using in vivo models. This may lead to the discovery of novel natural compounds which can act as anticancer agents with better therapeutic efficacy and minimal side effects.

Finally clinical trials can also be attempted which will yield effective, economic and safe natural anticancer drugs. Such screening programs are likely to yield some new compounds which may themselves act as drug molecules or excellent leads for designing and synthesizing new, novel compounds which can be used for cancer treatment. It will also help the researcher in selecting a promising medicinal plant for in vivo studies and hence hasten the speed of exploiting the nature for anticancer drugs.

\section{Acknowledgements}

The authors thank Department of Biosciences (UGC-CAS) for providing excellent research facilities.

\section{References}

Abdullah AS, Mohammed AS, Abdullah R, Mirghani ME, Al-Qubaisi M (2014). Cytotoxic effects of Mangifera indica L. kernel extract on human breast cancer (MCF7 and MDA-MB-231 cell lines) and bioactive constituents in the crude extract. BMC
Complementary and Alternative Medicine. 14(1):199-209.

Ahamed A, Panneerselvam A, Alaklabi A, Arif IA, Ambikapathy V, Thajuddin N (2020). Molecular perspective and anticancer activity of medicinal plants. Saudi Journal of Biological Sciences. 27: 666-675.

Ali AQ, Farah MA, Abou-Tarboush FM, Al-Anazi KM, Ali MA, Lee J, Hailan WA, Mahmoud AH (2019). Cytogenotoxic effects of Adenium obesum seeds extracts on breast cancer cells. Saudi Journal of Biological Sciences. 26(3):547-553.

Al-Oqail MM, Farshori NN, Al-Sheddi ES, Musarrat J, Al-Khedhairy AA, Siddiqui MA (2013). In vitro cytotoxic activity of seed oil of fenugreek against various cancer cell lines. Asian Pacific Journal of Cancer Prevention. 14(3):1829-1832.

AlSalhi MS, Elangovan K, Ranjitsingh AJ, Murali P, Devanesan S (2019). Synthesis of silver nanoparticles using plant derived 4-N-methyl benzoic acid and evaluation of antimicrobial, antioxidant and antitumor activity. Saudi Journal of Biological Sciences. 26: 970-978.

Al-Sheddi ES, Farshori NN, Al-Oqail MM, Musarrat J, Al-Khedhairy AA, Siddiqui MA (2014). Cytotoxicity of Nigella sativa seed oil and extract against human lung cancer cell line. Asian Pacific Journal of Cancer Prevention. 15(2):983-987.

Amaral RG, dos Santos SA, Andrade LN, Severino P, Carvalho AA (2019). Natural Products as Treatment against Cancer: A Historical and Current Vision. Clinics in Oncology. 4(5).

Ascar IF, Al-A'Araji SB, Alshanon AF (2019). Cytotoxicity and antioxidant effect of ginger gold nanoparticles on thyroid carcinoma cells. Journal of Pharmaceutical Sciences and Research. 11(3):1044-1051.

Beltrán JMG, Espinosa C, Guardiola FA, Esteban MÁ (2018). In vitro effects of Origanum vulgare leaf extracts on gilthead seabream (Sparus aurata L.) leucocytes, cytotoxic, bactericidal and antioxidant activities. Fish \& Shellfish Immunology. 79:1-10.

Bibi Y, Nisa S, Zia M, Waheed A, Ahmed S, Chaudhary $\mathrm{MF}(2012)$. In vitro cytotoxic activity of Aesculus indica against breast adenocarcinoma cell line (MCF-7) and phytochemical analysis. Pakistan Journal of Pharmaceutical Sciences. 25(1): 183-187

Boutennoun H, Boussouf L, Rawashdeh A, Al- 
Qaoud K, Abdelhafez S, Kebieche M, Madani $\mathrm{K}$ (2017). In vitro cytotoxic and antioxidant activities of phenolic components of Algerian Achillea odorata leaves. Arabian Journal of Chemistry. 10(3):403-409.

Chanda S and Nagani K (2013). In vitro and in vivo methods for anticancer activity evaluation and some Indian medicinal plants possessing anticancer properties: an overview. Journal of pharmacognosy and Phytochemistry. 2(2):140-152.

Chang WL, Cheng FC, Wang SP, Chou ST, Shih Y (2017). Cinnamomum cassia essential oil and its major constituent cinnamaldehyde induced cell cycle arrest and apoptosis in human oral squamous cell carcinoma HSC- 3 cells. Environmental Toxicology. 32(2):456-68.

Chen L, Batjikh I, Hurh J, Han Y, Huo Y, Ali H, Li JF, Rupa EJ, Ahn JC, Mathiyalagan R, Yang DC(2019). Green synthesis of zinc oxide nanoparticles from root extract of Scutellaria baicalensis and its photocatalytic degradation activity using methylene blue. Optik. 184:324-329.

Devaraj P, Kumari P, Aarti C, Renganathan A (2013). Synthesis and characterization of silver nanoparticles using cannonball leaves and their cytotoxic activity against MCF-7 cell line. Journal of Nanotechnology. http://doi.org/10.1155/2013/598328.

Devi PU, Rao BS, Solomon FE (1998). Effect of plumbagin on the radiation induced cytogenetic and cell cycle changes in mouse Ehrlich ascites carcinoma in vivo. Indian Journal of Experimental Biology. 36(9):891895.

Diaz-Moralli S, Tarrado-Castellarnau M, Miranda A, Cascante M (2013). Targeting cell cycle regulation in cancer therapy. Pharmacology \& Therapeutics. 138:255-271.

Donga S, Pande J, Moteriya P and Chanda S (2017). In vitro cytotoxicity study of leaf, stem and bark of Pterocarpus santalinus Linn. f. Journal of Phrmacognosy and Phytochemistry. 6(4):297-305

Elshafie H, Armentano M, Carmosino M, Bufo S, De Feo V, Camele I (2017). Cytotoxic activity of Origanum vulgare $\mathrm{L}$. on hepatocellular carcinoma cell line HepG2 and evaluation of its biological activity. Molecules. 22(9):1435-1451.

Erdogan O, Abbak M, Demirbolat GM, Birtekocak F, Aksel M, Pasa S, Cevik O(2019). Green synthesis of silver nanoparticles via Cynara scolymus leaf extracts: The characterization, anticancer potential with photodynamic therapy in MCF7 cells. PloS One. 14(6), https://doi.org/10.1371/journal.pone.0216496.

Farooq M, Abutaha N, Mahboob S, Baabbad A, Almoutiri ND, Wadaan MA(2020). Investigating the antiangiogenic potential of Rumex vesicarius (humeidh), anticancer activity in cancer cell lines and assessment of developmental toxicity in Zebrafish embryos. Saudi Journal of Biological Sciences. 27(2):611-622.

Finco FDBA, Kloss L, Graeve L (2016). Bacaba (Oenocarpus bacaba) phenolic extract induces apoptosis in the MCF-7 breast cancer cell line via the mitochondria-dependent pathway. NFS Journal. 5:5-15.

Fronza M, Murillo R, Ślusarczyk S, Adams M, Hamburger M, Heinzmann B, Laufer S, Merfort I (2011). In vitro cytotoxic activity of abietane diterpenes from Peltodon longipes as well as Salvia miltiorrhiza and Salvia sahendica. Bioorganic \& Medicinal Chemistry. 19(16):4876-4881.

Gali-Muhtasib H and Bakkar N (2002). Modulating cell cycle: current applications and future prospects for future drug development. Current Cancer Drug Targets. 2, 1-17.

Gautam V, Sharma A, Arora S, Bhardwaj R, Ahmad A, Ahamad B, Ahmad P (2020). In-Vitro antioxidant, antimutagenic and cancer cell growth inhibition activities of Rhododendron arboreum leaves and flowers. Saudi Journal of Biological Sciences https://doi.org/10.1016/j.sjbs.2020.01.030.

GBD (2015). Disease and Injury Incidence and Prevalence Collaborators (2016). Global, regional and National incidence, prevalence and years lived with disability for 310 diseases and injuries, 1990-2015; a systematic analysis for the global burden on disease study, Lancet. 388:1545-1602.

George VC, Kumar DR, Rajkumar V, Suresh PK, Kumar RA (2012). Quantitative assessment of the relative antineoplastic potential of the nbutanolic leaf extract of Annona muricata Linn. in normal and immortalized human cell lines. Asian Pacific Journal of Cancer Prevention. 13(2):699-704.

Ghagane SC, Puranik SI, Kumbar VM, Nerli RB, Jalalpure SS, Hiremath MB, Neelagund S, Aladakatti R (2017). In vitro antioxidant and 
anticancer activity of Leea indica leaf extracts on human prostate cancer cell lines. Integrative Medicine Research. 6(1):79-87.

Ghasemzadeh A, Karbalaii MT, Jaafar HZ, Rahmat A (2018). Phytochemical constituents, antioxidant activity, and antiproliferative properties of black, red, and brown rice bran. Chemistry Central Journal. 12(1):1-13.

Gibellini L, Pinti M, Nasi M, De Biasi S, Roat E, Bertoncelli L, Cossarizza A (2010). Interfering with ROS metabolism in cancer cells: the potential role of quercetin. Cancers 2: $1288-1311$.

Goh BH and Kadir AH (2011). In vitro cytotoxic potential of Swietenia macrophylla King seeds against human carcinoma cell lines. Journal of Medicinal Plants Research. 5(8):1395-1404.

Gomathi AC, Rajarathinam SX, Sadiq AM, Rajeshkumar S (2020). Anticancer activity of silver nanoparticles synthesized using aqueous fruit shell extract of Tamarindus indica on MCF-7 human breast cancer cell line. Journal of Drug Delivery Science and Technology. 155 : https://doi.org/10.1016/j.jddst.2019.101376.

Gopinath V, Priyadarshini S, MubarakAli D, Loke MF, Thajuddin N, Alharbi NS, Yadavalli T, Alagiri M, Vadivelu J (2016). AntiHelicobacter pylori, cytotoxicity and catalytic activity of biosynthesized gold nanoparticles: Multifaceted application. Arabian Journal of Chemistry. https://doi.org/10.1016/j.arabjc.2016.02.005.

Graidist P, Martla M, Sukpondma Y (2015). Cytotoxic activity of Piper cubeba extract in breast cancer cell lines. Nutrients. 7(4):27072718.

Greenwell M and Rahman PK (2015). Medicinal plants: their use in anticancer treatment. International Journal of Pharmaceutical Sciences and Research. 6(10):4103-4112.

Hassan HM, Mina SA, Bishr MM, Khalik SM (2018). Influence of foliar spray of ethephon and water stress on the essential oil composition and impact on the cytotoxic activity of Thymus vulgaris aerial parts. Natural Product Research. https://doi.org/ 10.1080/ 14786419.2018.1460843.

Hassanien R, Husein DZ, Al-Hakkani MF (2018). Biosynthesis of copper nanoparticles using aqueous Tilia extract: antimicrobial and anticancer activities. Heliyon. 4(12): https://doi:10.1016/j.heliyon.2018.e01077.

He Y, Wei F, Ma Z, Zhang H, Yang Q, Yao B, Huang Z, Li J, Zeng C, Zhang Q(2017). Green synthesis of silver nanoparticles using seed extract of Alpinia katsumadai, and their antioxidant, cytotoxicity, and antibacterial activities. RSC Advances. 7(63):3984239851.

Hu Y, Zhou J, Ye F, Xiong H, Peng L, Zheng Z, Xu F, Cui M, Wei C, Wang X, Wang Z (2015). BRD4 inhibitor inhibits colorectal cancer growth and metastasis. International Journal of Molecular Sciences. 16(1):1928-1948.

Iqbal J, Abbasi BA, Mahmood T, Kanwal S, Ali B, Shah SA, Khalil AT (2017). Plant-derived anticancer agents: A green anticancer approach. Asian Pacific Journal of Tropical Biomedicine. 7(12):1129-1150.

Jamdagni P, Khatri P, Rana JS(2018). Green synthesis of zinc oxide nanoparticles using flower extract of Nyctanthes arbor-tristis and their antifungal activity. Journal of King Saud University-Science. 30(2):168-175.

Karthika V, Arumugam A, Gopinath K, Kaleeswarran P, Govindarajan M, Alharbi NS, Kadaikunnan S, Khaled JM, Benelli G(2017). Guazuma ulmifolia barksynthesized $\mathrm{Ag}, \mathrm{Au}$ and $\mathrm{Ag} / \mathrm{Au}$ alloy nanoparticles: Photocatalytic potential, DNA/protein interactions, anticancer activity and toxicity against 14 species of microbial pathogens. Journal of Photochemistry and Photobiology B: Biology.167:189-99.

Khara G, Padalia H, Moteriya P, Chanda S (2018). Peltophorum pterocarpum flower-mediated synthesis, characterization, antimicrobial and cytotoxic activities of $\mathrm{ZnO}$ nanoparticles. Arabian Journal for Science and Engineering. 43(7):3393-3401.

Liu M, Li XQ, Weber C, Lee CY, Brown J, Liu RH (2002). Antioxidant and antiproliferative activities of raspberries. Journal of Agricultural and Food Chemistry. 50(10):2926-2930.

Luo H, Cai Y, Peng Z, Liu T, Yang S (2014). Chemical composition and in vitro evaluation of the cytotoxic and antioxidant activities of supercritical carbon dioxide extracts of pitaya (dragon fruit) peel. Chemistry Central Journal. 8(1):1-7.

Malar T R J J, Antonyswamy J, Vijayaraghavan P, Kim YO, Al-Ghamdi AA, Elshikh MS, Hatamleh AA, Al-Dosary MA, Na SW, Kim 
HJ (2020). In vitro phytochemical and pharmacological bio-efficacy studies on Azadirachta indica A. Juss and Melia azedarach Linn. for anticancer activity. Saudi Journal of Biological Sciences. 27: 682-688.

Malek SN, Phang CW, Ibrahim H, Abdul Wahab N, Sim KS (2011). Phytochemical and cytotoxic investigations of Alpinia mutica rhizomes. Molecules. 16(1):583-589.

Maragheh AD, Tabrizi MH, Karimi E, Seyedi SM, Khatamian N (2019). Producing the sour cherry pit oil nanoemulsion and evaluation of its anti-cancer effects on both breast cancer murine model and MCF-7 cell line. Journal of Microencapsulation. 36(4):399-409.

Mavundza EJ, Tshikalange TE, Lall N, Hussein AA, Mudau FN, Meyer JJM (2010). Antioxidant activity and cytotoxicity effect of flavonoids isolated from Athrixia phylicoides. Journal of Medicinal Plants Research 4: 2584-2587.

Meera R and Chidambaranathan N (2017). Anti cancer activity of ethanolic extract of Crataeva magna Lour (DC) against Ehrlich ascitic carcinoma cell lines in mice. Journal of Pharmaceutical Sciences and Research. 9(10):1869-1873.

Mishra A, Sharma AK, Kumar S, Saxena AK, Pandey AK (2013). Bauhinia variegata leaf extracts exhibit considerable antibacterial, antioxidant, and anticancer activities. BioMed Research International. http://dx.doi.org/10.1155/2013/915436.

Mohammadi-Aloucheh R, Habibi-Yangjeh A, Bayrami A, Latifi-Navid S, Asadi A (2018). Enhanced anti-bacterial activities of $\mathrm{ZnO}$ nanoparticles and $\mathrm{ZnO} / \mathrm{CuO}$ nanocomposites synthesized using Vaccinium arctostaphylos L. fruit extract. Artificial cells, Nanomedicine, and Biotechnology. 46(1):1200-1209.

Mollick MM, Rana D, Dash SK, Chattopadhyay S, Bhowmick B, Maity D, Mondal D, Pattanayak S, Roy S, Chakraborty M, Chattopadhyay D (2015). Studies on green synthesized silver nanoparticles using Abelmoschus esculentus (L.) pulp extract having anticancer (in vitro) and antimicrobial applications. Arabian Journal of Chemistry. https://doi.org/ 10.1016/j.arabjc.2015.04.033.

Mollick MMR, Rana D, Dash SK, Chattopadhyay S, Bhowmick B, Maity D, Mondal D, Pattanayak S, Roy S, Chakraborty M, Chattopadhyay $\mathrm{D}(2015)$. Studies on green synthesized silver nanoparticles using Abelmoschus esculentus (L.) pulp extract having anticancer (in vitro) and antimicrobial applications. Arabian Journal of Chemistry. 12: 2572-2584.

Moteriya P and Chanda S (2017). Synthesis and characterization of silver nanoparticles using Caesalpinia pulcherrima flower extract and assessment of their in vitro antimicrobial, antioxidant, cytotoxic, and genotoxic activities. Artificial Cells, Nanomedicine, and Biotechnology.45(8):1556-1567.

Nagani K and Chanda S (2013). Antioxidant and anticancer activity of Cissus quadrangularis L. stem. LAP LAMBERT Academic Publishing GmbH \& Co. KG, HeinrichBocking-Straße 6-8, 66121 Saarbrucken, Germany, p.68.

Padalia H and Chanda S(2017). Characterization, antifungal and cytotoxic evaluation of green synthesized zinc oxide nanoparticles using Ziziphus nummularia leaf extract. Artificial cells, Nanomedicine, and Biotechnology. 45(8):1751-1761.

Patel PR, Nagar AA, Patel RC, Rathod DK, Patel VR (2011). In-vitro anticancer activity of Rubia Cordifolia against Hela and Hep-2 cell lines. International Journal of Pharmacy and Pharmaceutical Sciences. 2(3):44-46.

Patel RM and Patel SK (2011). Cytotoxic activity of methanolic extract of Artocarpus heterophyllus against A549, Hela and MCF-7 cell lines. Journal of Applied Pharmaceutical Science. 1(7):167-171.

Perera LMS, Veloz AIR, GoisRuiz ALT, Foglio MA, de Oliveira Sousa IM, Tinti SV, Possenti A, de Carvalho JE (2019). In vitro and in vivo antiproliferative activity of extracts and fractions of leaves and stem from Tabebuia hypoleuca (C. Wright) Urb. Drug Discovery. 13: 10-21.

Privitera G, Luca T, Castorina S, Passanisi R, Ruberto G, Napoli E (2019). Anticancer activity of Salvia officinalis essential oil and its principal constituents against hormonedependent tumour cells. Asian Pacific Journal of Tropical Biomedicine. 9(1):24-28.

Rajkuberan C, Prabukumar S, Sathishkumar G, Wilson A, Ravindran K, Sivaramakrishnan S (2017). Facile synthesis of silver nanoparticles using Euphorbia antiquorum L. latex extract and evaluation of their biomedical perspectives as anticancer agents. 
Journal of Saudi Chemical Society. 21(8):911-919.

Ruckmani A, Geetha L, Madhavi E (2015). Current scenario of cancer clinical trials in India. 9th Indo-Global summit on Cancer therapy, Hyderabad, India.

Russo A, Cardile V, Sanchez F, Troncoso N, Vanella A, Garbarino JA (2004). Chilean propolis: antioxidant activity and antiproliferative action in human tumor cell lines. Life Sciences. 76(5):545-558.

Saklani S, Kawra M, Parcha V(2019). Herbalderived anticancer phytoconstituents: tradition to molecular mechanisms. International Journal of Pharmaceutical Sciences and Research. 10(4):1632-1639.

Sani TA, Mohammadpour E, Mohammadi A, Memariani T, Yazdi MV, Rezaee R, Calina D, Docea AO, Goumenou M, Etemad L, Shahsavand S (2017). Cytotoxic and apoptogenic properties of Dracocephalum kotschyi aerial part different fractions on calu6 and mehr-80 lung cancer cell lines. Farmacia. 65(2):189-199.

Sarvmeili N, Jafarian-Dehkordi A, Zolfaghari B (2016). Cytotoxic effects of Pinus eldarica essential oil and extracts on HeLa and MCF-7 cell lines. Research in Pharmaceutical Sciences. 11(6):476- 483.

Sathyabama S and Sankaranarayanan S (2015). An in vitro biosynthesis of zinc oxide nanoparticles using rich flavonoid extract from the petals of Delonix regia and evaluation of their antioxidant and anticancer properties. International Journal of Pharmacognosy and Phytochemical Research. 7(5):1112-1119.

Seca AM, Pinto DC (2018). Plant secondary metabolites as anticancer agents: successes in clinical trials and therapeutic application. International Journal of Molecular Sciences. 19(1):263-268.

Shaban A, Mishra GM, Nautiyal R, Srivastava S, Tripathi K, Chaudhary P, Verma SK (2012). In vitro cytotoxicity of Moringa oleifera against different human cancer cell lines. Asian Journal of Pharmaceutical and Clinical Research 5(4): 271-272.

Shahneh F, Baradaran B, Majidi J, Babaloo Z (2014). Echinophora platyloba DC (Apiaceae) crude extract induces apoptosis in human prostate adenocarcinoma cells (PC 3). Biomedical Journal. 37(5):298-304.
Siegel RL, Miller KD, Jemal A (2016). Cancer statistics, 2016. CA: A Cancer Journal for Clinicians. 66(1):7-30.

Singh S, Ishar MP, Saxena AK, Kaur A (2013). Cytotoxic effect of Anthocephalus cadamba Miq. leaves on human cancer cell lines. Pharmacognosy Journal. 5(3):127-129.

Sirsat AP, Kokate PS, Shirsat RP (2019). Indian medicinal plants with potential antioxidant and anticancer properties: An insight. European Journal of Biotechnology and Bioscience. 7(1):47-51.

Sivaraj C, Saraswathi K, Arumugam P, Bhaskaran R, Manimaran A (2019). GC-MS analysis, antioxidant, antibacterial and anticancer activities of methanol bark extract of Albizia lebbeck (L.). The Journal of Phytopharmacology. 8(4): 177-184.

Stopeck AT and Thompson PA (2012). Breast cancer treatment and anagement. WebMD Health Professional Network Retrieved September. 292012, from medscape: <http://emedicine.medscape.com/article/1947 145-treatment.

Syed A, Benit N, Alyousef AA, Alqasim A, Arshad M (2020). In-vitro antibacterial, antioxidant potentials and cytotoxic activity of the leaves of Tridax procumbens. Saudi Journal of Biological Sciences. 27: 757-761.

Tejaputri NA, Arsianti A, Qorina F, Fithrotunnisa Q, Azizah NN, Putrianingsih R (2020). Anticancer activity of Ruellia britoniana flower on cervical HeLa cancer cells. Pharmacognosy Journal. 12(1): 29-34.

Tuama AA and Mohammed AA (2019). Phytochemical screening and in vitro antibacterial and anticancer activities of the aqueous extract of Cucumis sativus. Saudi Journal of Biological Sciences. 26(3):600604.

Turan I, Demir S, Kilinc K, Burnaz NA, Yaman SO, Akbulut K, Mentese A, Aliyazicioglu Y, Deger O (2017). Antiproliferative and apoptotic effect of Morus nigra extract on human prostate cancer cells. Saudi Pharmaceutical Journal. 25(2):241-248.

Turan I, Demir S, Kilinc K, Yaman SO, Misir S, Kara H, Genc B, Mentese A, Aliyazicioglu Y, Deger O (2018). Cytotoxic effect of Rosa canina extract on human colon cancer cells through repression of telomerase expression. Journal of Pharmaceutical Analysis. 8(6):394399. 
Wang H, Qiu C, Chen L, Abbasi AM, Guo X, Liu RH (2019). Comparative study of phenolic profiles, antioxidant and antiproliferative activities in different vegetative parts of ramie (Boehmeria nivea L.). Molecules. 24(8): https://doi:10.3390/molecules24081551.

Wang L, Xu J, Yan Y, Liu H, Karunakaran T, Li F (2019). Green synthesis of gold nanoparticles from Scutellaria barbata and its anticancer activity in pancreatic cancer cell (PANC- 1). Artificial cells, Nanomedicine, and Biotechnology. 47(1):1617-1627.

Wang L, Xu J, Yan Y, Liu H, Karunakaran T, Li F (2019). Green synthesis of gold nanoparticles from Scutellaria barbata and its anticancer activity in pancreatic cancer cell (PANC- 1). Artificial cells, Nanomedicine, and Biotechnology. 47(1):1617-1627.

Wang M, Chen Y, Hu P, Ji J, Li X, Chen J (2018). Neoclerodane diterpenoids from Scutellaria barbata with cytotoxic activities. Natural Product Research. https://doi.org/10.1080/14786419.2018.15143 99.

Weissenstein U, Kunz M, Urech K, Baumgartner S (2014). Interaction of standardized mistletoe (Viscum album) extracts with chemotherapeutic drugs regarding cytostatic and cytotoxic effects in vitro. BMC Complementary and Alternative Medicine. 14(1):4-9.

WHO (2016). Cancer fact sheets (http://www.who.int/cancer/en/; Assessed on 31st July 2017).

Yang C, Chen H, Chen H, Zhong B, Luo X, Chun J (2017). Antioxidant and anticancer activities of essential oil from Gannan navel orange peel. Molecules. 22(8):1-10.

Yuan CG, Huo C, Gui B, Cao WP (2016). Green synthesis of gold nanoparticles using Citrus maxima peel extract and their catalytic/antibacterial activities. IET Nanobiotechnology. 11(5):523-530.

Yugandhar P, Vasavi T, Devi PU, Savithramma N (2017). Bioinspired green synthesis of copper oxide nanoparticles from Syzygium alternifolium (Wt.) Walp: characterization and evaluation of its synergistic antimicrobial and anticancer activity. Applied Nanoscience. 7(7):417-427.

Zhong Z, Yu H, Wang S, Wang Y, Cui L (2018). Anti-cancer effects of Rhizoma curcumae against doxorubicin-resistant breast cancer cells. Chinese Medicine. 13(1):1-0.

Zhou JX, Braun MS, Wetterauer P, Wetterauer B, Wink M (2019). Antioxidant, cytotoxic, and antimicrobial activities of Glycyrrhiza glabra L., Paeonia lactiflora Pall., and Eriobotrya japonica (Thunb.) Lindl. extracts. Medicines. 6(43): 1-16.

Zubair MS, Anam S, Lallo S (2016). Cytotoxic activity and phytochemical standardization of Lunasia amara Blanco wood extract. Asian Pacific Journal of Tropical Biomedicine. 6(11):962-966.

\section{How to cite this article:}

Jyoti Pande and Sumitra Chanda. 2020. Screening of Anticancer Properties of some Medicinal Plants - Review. Int.J.Curr.Microbiol.App.Sci. 9(03): 1348-1362.

doi: https://doi.org/10.20546/ijcmas.2020.903.157 